\title{
Silica-Based Highly Nonlinear Fibers with a High SBS Threshold
}

\author{
Lars Grüner-Nielsen ${ }^{1}$, Søren Herstrøm ${ }^{1}$, Sonali Dasgupta ${ }^{2}$, David Richardson ${ }^{2}$, Dan Jakobsen ${ }^{1}$, Carl \\ Lundström $^{3}$, Peter A. Andrekson ${ }^{3}$, Martin E. V. Pedersen ${ }^{1}$, and Bera Pálsdóttir ${ }^{1}$ \\ 1: OFS Fitel Denmark ApS, Priorparken 680, DK-2605 Brøndby, Denmark, lgruner@ ofsoptics.com \\ 2:Optoelectronics Research Centre, University of Southampton, United Kingdom SO17 1BJ \\ 3: Photonics Laboratory, Department of Microtechnology and Nanoscience, Chalmers University of \\ Technology, Gothenburg, Sweden
}

\begin{abstract}
Results on suppression of stimulated Brillouin scattering in highly non linear fibers with germanium-doped core as well as aluminum-doped core based on spooling the fiber with a linear strain gradient is presented.
\end{abstract}

\section{INTRODUCTION}

A serious limitation in many applications of Highly Non Linear Fibers (HNLF) is Stimulated Brillouin Scattering (SBS), which limits the amount of power that can be launched into the fiber. This, in turn, limits the maximum nonlinearity that can be obtained from a HNLF, which scales as:

$$
\gamma \mathrm{L}_{\text {eff }} \mathrm{P}_{\mathrm{th}} \propto \frac{\mathrm{n}_{2}}{\mathrm{~g}_{\mathrm{B}}},
$$

where $\gamma$ is the nonlinear coefficient, $\mathrm{L}_{\text {eff }}$ is the effective length, $\mathrm{P}_{\text {th }}$ is the threshold power required for the onset of $\mathrm{SBS}, \mathrm{n}_{2}$ is the nonlinear refractive index, and $\mathrm{g}_{\mathrm{B}}$ the Brillouin gain coefficient of the fiber. (1) is often referred to as the figure of merit for SBS limited HNLFs [1].

Several methods have been proposed for increasing the SBS threshold: Linear or stepwise temperature gradient along the HNLF [2], linear or stepwise strain gradient along the fiber [3], and finally doping the core with aluminum instead of germanium [4,5]. In this paper we will report on the effect of straining on Ge-doped and Al-doped HNLF.

\section{RESULTS}

SBS suppression by straining the HNLF is obtained because the SBS gain peak is shifted in wavelength when the fiber is strained. So, by spooling the HNLF with a linear straingradient along its length the SBS gain spectrum can be broadened and the SBS threshold consequently increased. A drawback of applying tension is that the tension also changes the dispersion properties of the HNLF. The measured effect of spool tension on both SBS shift and dispersion for a $125 \mu \mathrm{m}$ cladding diameter Ge-doped HNLF is shown in Figure 1. A change in SBS shift of $290 \mathrm{MHz} / \%$ and a change in dispersion of $-0.65 \mathrm{ps} /(\mathrm{nm} \cdot \mathrm{km}) / \%$ is found (using that a $1000 \mathrm{~g}$ fiber tension gives a fiber elongation of $1.1 \%$ ). This is in good agreement with the findings of Takahashi et al., who found a change in SBS shift of $340 \mathrm{MHz} / \%$ and a change in dispersion of $-0.7 \mathrm{ps} /(\mathrm{nm} \cdot \mathrm{km}) / \%$ for a germanium doped HNLF with $50 \mu \mathrm{m}$ cladding diameter [6].

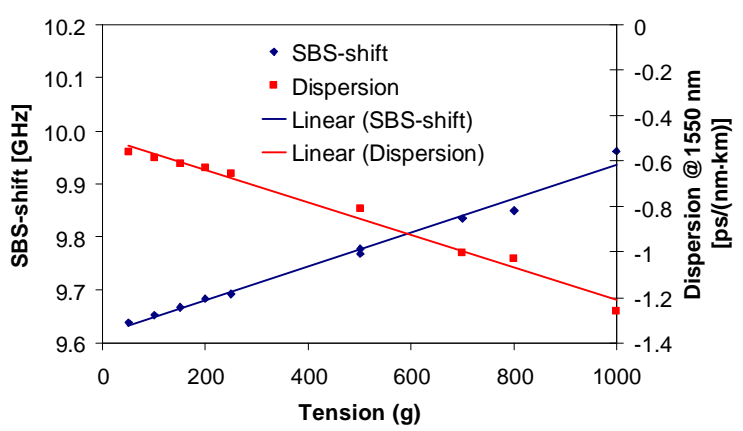

Figure 1. Measured change in SBS frequency shift and dispersion versus spooling tension for a Ge-doped HNLF. Also shown are linear fits to the measured data.

A $300 \mathrm{~m}$ HNLF module has been made on a spool with $200 \mathrm{~mm}$ core where the tension is linearly ramped from 1000 $\mathrm{g}$ to 0 . Measured Stokes spectra before and after straining are shown in Figure 2.

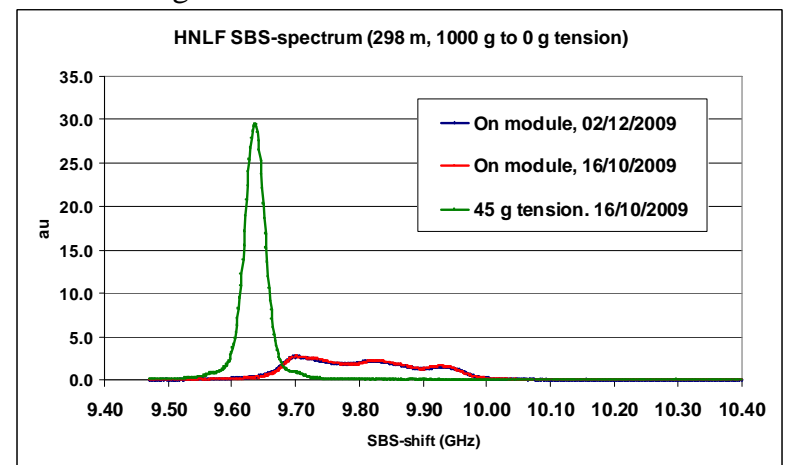

Figure 2. Measured spectra of Stokes light for $298 \mathrm{~m}$ Ge-doped HNLF before and after straining

It is observed from Figure 2 that the SBS spectrum is broadened by a factor of 8.5 from a FWHM value of $34 \mathrm{MHz}$ to $290 \mathrm{MHz}$. It can also be seen that the straining remains stable over $1 \frac{1}{2}$ month. When comparing the spectrum for the strained fiber with the spectrum for the unstrained fiber in Figure 2, with the in Figure 1 found relation between SBS 
shift and strain, it looks like the tension is effectively only

ramped between 1000 and $150 \mathrm{~g}$.

Table 1. Measured properties for Ge-doped and Al-doped HNLF spooled with low tension and with a linear strain gradient where tension is decreased linearly from 1000 to $0 \mathrm{~g}$.

\begin{tabular}{|c|c|c|c|c|c|}
\hline & & \multicolumn{2}{|c|}{ Ge-doped HNLF } & \multicolumn{2}{|c|}{ Al doped HNLF } \\
\hline & & Before straining & After straining & Before straining & After straining \\
\hline Length & $\mathrm{m}$ & \multicolumn{2}{|c|}{298} & \multicolumn{2}{|c|}{179} \\
\hline Fiber attenuation @ 1550 nm & $\mathrm{dB} / \mathrm{km}$ & \multicolumn{2}{|c|}{0.83} & \multicolumn{2}{|c|}{15} \\
\hline Effective area@1550 nm & $\mu \mathrm{m}^{2}$ & \multicolumn{2}{|c|}{11.5} & \multicolumn{2}{|c|}{13.5} \\
\hline Non linear coefficient & $(\mathrm{W} \cdot \mathrm{km})^{-1}$ & \multicolumn{2}{|c|}{11.6} & \multicolumn{2}{|c|}{7.4} \\
\hline Dispersion@1550 nm & $\mathrm{ps} /(\mathrm{nm} \cdot \mathrm{km})$ & 0.22 & -0.06 & -0.12 & -0.35 \\
\hline Dispersion slope @ 1550 nms & $\mathrm{ps} /\left(\mathrm{nm}^{2} \cdot \mathrm{km}\right)$ & 0.018 & 0.018 & 0.011 & 0.011 \\
\hline Zero dispersion wavelength & $\mathrm{nm}$ & 1538 & 1553 & 1562 & 1598 \\
\hline PMD & ps & 0.05 & 0.31 & 0.10 & 0.50 \\
\hline SBS frequency shift & $\mathrm{GHz}$ & 9.63 & 9.82 & 11.71 & 11.90 \\
\hline SBS FWHM spectral width & $\mathrm{MHz}$ & 34 & 290 & 52 & 245 \\
\hline $\mathrm{P}_{\text {th }} \cdot \mathrm{L}_{\mathrm{eff}}$ & $\mathrm{W} \cdot \mathrm{m}$ & 18.3 & 82 & 85 & 211 \\
\hline $\mathrm{P}_{\mathrm{th}} \cdot \gamma \cdot \mathrm{L}_{\mathrm{eff}}$ & & 0.21 & 0.93 & 0.63 & 1.56 \\
\hline
\end{tabular}

A summary of all measurements on strained and unstrained Ge-doped HNLF as well as an Al-doped HNLF [5] is presented in Table 1. The SBS threshold $\left(\mathrm{P}_{\text {th }}\right)$ is defined as the input pump power where the reflected power is equal to $1 \%$ of the input power. Stokes spectra before and after straining of $179 \mathrm{~m} \mathrm{Al-doped} \mathrm{HNLF}$ is shown in Figure 3.

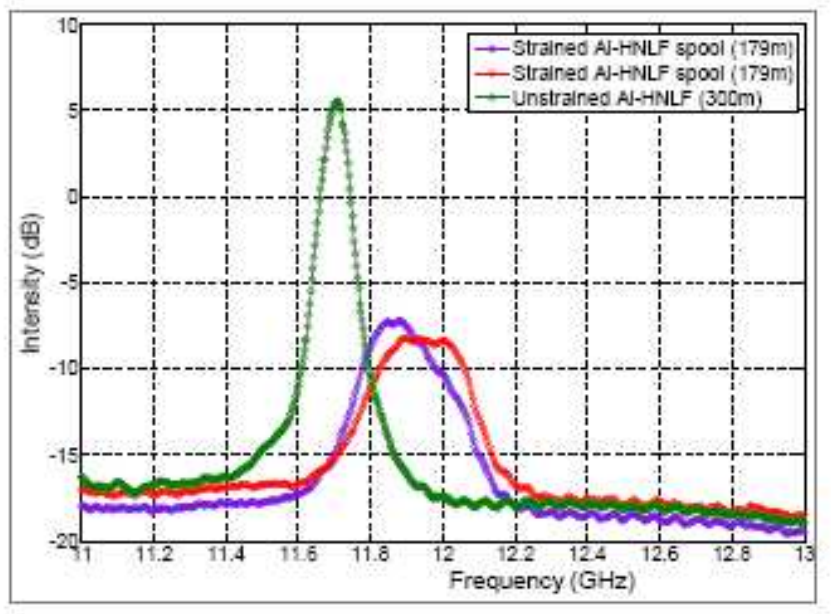

Figure 3. Measured spectra of Stokes light for $170 \mathrm{~m}$ Al-doped HNLF before and after straining.

It is observed that the change in SBS frequency shift due to straining is $190 \mathrm{MHz}$ for both the Ge-doped and Al-doped fibers, thus showing that the SBS shift versus strain it the same for the Al-doped HNLF as for the Ge-doped HNLF. The change in dispersion due to straining is slightly higher for the Ge-doped HNLF $(0.28 \mathrm{ps} /(\mathrm{nm} \cdot \mathrm{km}))$ than for the Al-doped HNLF $(0.23 \mathrm{ps} /(\mathrm{nm} \cdot \mathrm{km}))$. However, due to lower dispersion slope of the Al-doped HNLF, the change in its ZDW is larger than for the Ge-doped HNLF. PMD is observed to increase significantly due to the straining. This is attributed to stress induced birefringence that arises due to the pressure that the fiber experiences at the core of the spool. This effect could be diminished by using a larger spool size. A larger improvement in SBS threshold is obtained for the Ge-doped HNLF (6.5 dB) as compared to the Al-doped HNLF (4.0 dB) for the same straining level due to the smaller spectral width of the SBS spectrum for the Ge-doped HNLF.

Finally, it is noted that the Al-doped HNLF has compared to the Ge-doped HNLF the advantage of $6.7 \mathrm{~dB}$ larger SBS threshold, and due to lower non-linear coefficient, a $4.8 \mathrm{~dB}$ larger FOM. The Al-doped HNLF has the disadvantage of higher attenuation coefficient.

\section{CONCLUSION}

Spooling of HNLF with a linear strain gradient is an efficient method to increase the SBS threshold. Compared to applying a temperature gradient, which typical gives a SBS shift of $0.5 \mathrm{MHz} /{ }^{\circ} \mathrm{C}$ [7], a temperature gradient of $380^{\circ} \mathrm{C}$ would be necessary to get the same SBS suppression as a tension gradient from 1000 to $0 \mathrm{~g}$. Also spooling the fiber with a strain gradient is much easier to implement in practice than a permanent temperature gradient. However, straining has the draw back of dispersion change, increased PMD, as well as long term reliability issues due to the high permanent strain.

\section{ACKNOWLEDGEMENT}

This research has received funding from the European Communities Seventh Framework Program FP/2007-2013 under grant agreement 224547 (PHASORS).

\section{REFERENCES}

1 J. H. Lee et al., Optics Letters 30, 1698 (2005)

2 J. Hansryd et al., Journal of Lightwave Technology 19, 1691 (2001)

3 J. M. Chavez Boggio et al., Journal of Lightwave Technology 23, 3808 (2005)

4 T. Nakasinishi et al., Proceedings of ECOC'06, Th4.2.2 (2006)

5 L. Grüner-Nielsen et al., Proceedings of ECOC'10, Tu.4.D.3 (2010),

6 M. Takahashi et al.; Journal of Lightwave Technology 83, 59 (2010)

7 M. R. Lorenzen et al., Electronics Letters 45, 125 (2009) 